\title{
DTI Findings Correlated with Neurocognitive Impairments in Basal Ganglia Stroke: A Preliminary Study
}

Kwang-lk Jung ${ }^{1}$, Ah-Young Jun ${ }^{3}$, Yun-Hee Kim ${ }^{4}$, Shahid Bashir ${ }^{5}$, Suk Hoon Ohn ${ }^{1}$, SeungHo Ahn ${ }^{1}$, Chang-hyun Park ${ }^{4}$ and Woo-Kyoung Yoo ${ }^{1,2^{*}}$

${ }^{1}$ Department of Physical Medicine and Rehabilitation, Hallym University Sacred Heart Hospital, Republic of Korea

${ }^{2}$ Hallym Institute for Translational Genomics \& Bioinformatics, Hallym University College of Medicine

${ }^{3}$ Department of Physical Medicine and Rehabilitation, Hallym University Dongtan Sacred Heart Hospital, Republic of Korea

${ }^{4}$ Department of Physical Medicine and Rehabilitation, Samsung Medical Center, Sungkyunkwan University School of Medicine, Republic of Korea

${ }^{5}$ Department of Physiology, Faculty of Medicine, King Saud University, Saudi Arabia

${ }^{6}$ Berenson-Allen Center for Noninvasive Brain Stimulation, Beth Israel Deaconess Medical Center, Harvard Medical School, USA

"Corresponding author: Woo-Kyoung Yoo, Department of Physical Medicine and Rehabilitation, Hallym University College of Medicine, Republic of Korea; Tel: 82-31-380-1875; Fax: 82-31-380-3864; E-mail: wooky@hallym.ac.kr

Received date: June 08, 2015; Accepted date: June 26, 2015; Published date: June 30, 2015

Copyright: $\odot 2015$ Jung K, et al. This is an open-access article distributed under the terms of the Creative Commons Attribution License, which permits unrestricted use, distribution, and reproduction in any medium, provided the original author and source are credited.

\begin{abstract}
s
Objective: Subcortical lesion involving basal ganglia can cause a wide range of behavioral and cognitive alterations largely attributed to disconnection of subcortical fibers; however, the underlying neural correlates of cognitive dysfunction based on the integrity of the white matter fiber has not yet been explored in stroke patients.
\end{abstract}

Methods: The correlations among cognitive function and diffusion metrics of each subcortical fiber were investigated in nine basal ganglia stroke patients by diffusion tensor imaging (DTI) in our study.

Results: Patient's general cognitive and verbal working memory function correlated well with the integrity of the left superior longitudinal fasciculus (SLF), nonverbal attention with the right inferior longitudinal fasciculus (ILF), SLF, and frontostriatal (FS) fibers. On the other hand, nonverbal long-term memory correlated well with the integrity of the right ILF and FS fibers.

Conclusions: Neurocognitive characteristics of patients with basal ganglia stroke seem to reflect the integrity of subcortical fiber connectivity.

Keywords: Disconnection; Subcortical connectivity; Neuropsychological test; Subcortical stroke; White matter integrity

\section{Introduction}

Previous studies have demonstrated that the basal ganglia mediates not only motor function, but also cognitive functions [1,2]. Lesion studies revealed a wide range of behavioral and cognitive alterations, including impairment of memory, attention and executive functions [3,4], aphasia [5], and neglect after damage to the basal ganglia [3]. Most of these reports were based on clinical observation rather than systematic assessment of anatomical substrates.

Basal ganglia are centrally located subcortical structure with many subcortical fiber bundles traverse near or around these structures. Thus, disconnection syndrome caused by damage to subcortical fiber connectivity presumed to cause various cognitive dysfunctions. Conventional MR imaging cannot provide reliable information about the integrity of white matter tracts, thereby limiting the ability to predict clinical outcome. Recent advances in diffusion tensor imaging (DTI) [6] have allowed in vivo investigation of subcortical fiber integrity.

Previous DTI studies related to cognitive impairments in stroke have been mostly focused on small vessel disorders [7-9]. To the best of our knowledge there were no studies published aimed to delineate the correlation of the neuropsychological impairments and DTI metrics purely in moderate to large lesion in basal ganglia. There were one recently published study that showed the predictability of cognitive impairments in ischemic stroke using DTI, however, they also included subjects of cortical and subcortical lesion [10].

The aim of this study was to unravel the relationship among cognitive function and subcortical fiber connectivity using DTI and fiber tractography in subcortical stroke patients.

\section{Methods}

\section{Subjects}

Nine patients who had first stroke in basal ganglia participated in this study. Three patients and six patients were diagnosed as basal ganglia infarction and basal ganglia hemorrhage in each. Five patients had lesions in the left hemisphere while four patients had lesion in the right hemisphere (Figure 1). Exclusion criteria consisted of (1) history of pre-stroke cognitive impairment and other neurologic or severe systemic disease (2) history of Parkinson's disease, alcohol abuse, or psychiatric illness that might compromise cognitive function, (3) who have aphasia or unavailability for neurocognitive function test, (4) who have significant abnormal brain lesion in brain MRI study other 
Citation: Jung K, Jun A, Kim Y, Bashir S, Ohn SH, et al. (2015) DTI Findings Correlated with Neurocognitive Impairments in Basal Ganglia Stroke: A Preliminary Study. J Neurol Neurophysiol 6: 299. doi:10.4172/2155-9562.1000299

Page 2 of 5

than basal ganglia, (5) basal ganglia lesion secondary to head injury, coagulation abnormalities, or any secondary cause of hemorrhage such as cerebral aneurysm. The subjects' handedness was assessed using the Edinburgh Handedness Inventory and all subjects were deemed righthanded [11]. The patients' mean age was $55.3 \pm 9.7$ years. Mean duration from stroke onset to DTI assessment was 5.9 months. The degree of disability measured by modified Rankin Scale was $2.89 \pm$ 1.27. All participants were provided written informed consent.

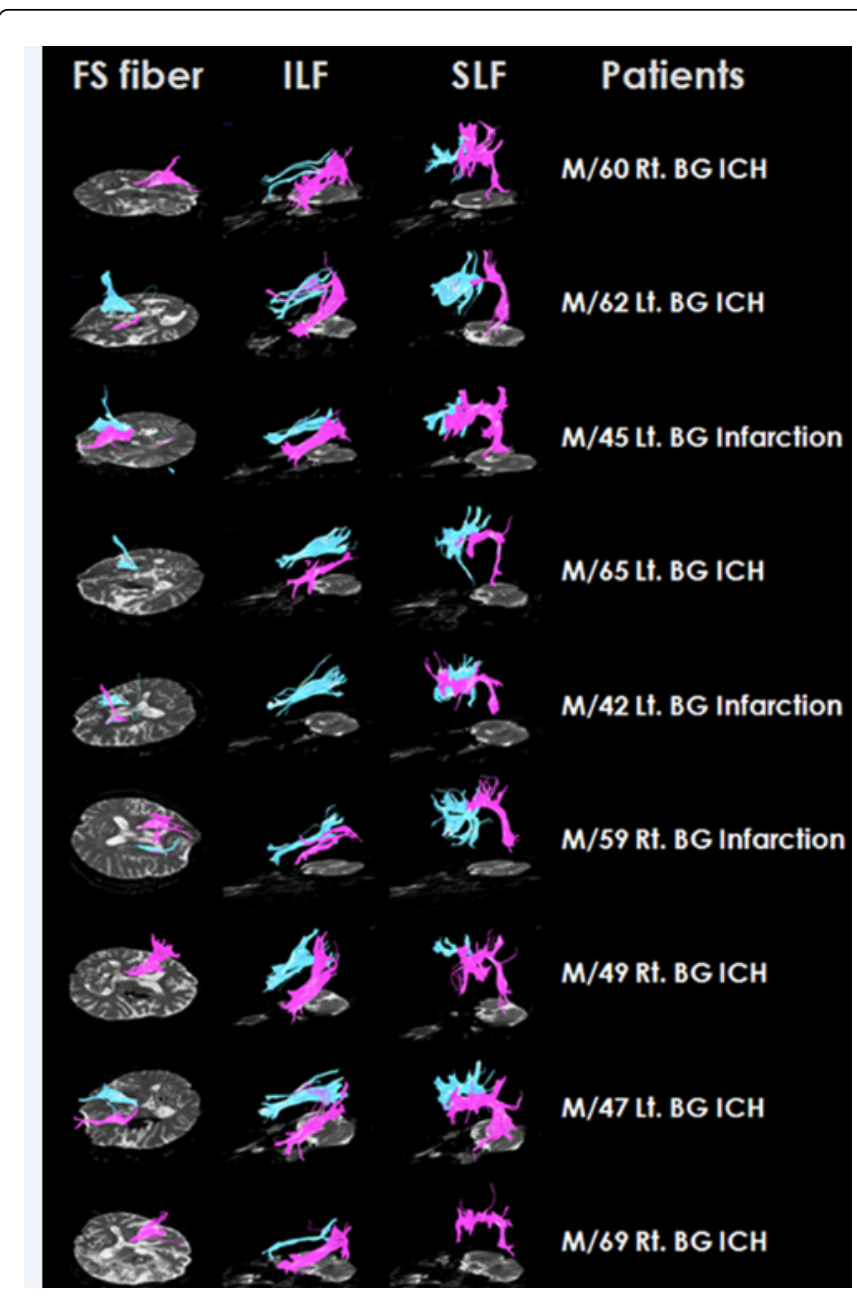

Figure 1: Subcortical fiber tract obtained by diffusion tensor tractography in nine basal ganglia stroke patients. Pink color represents right side, cyan blue color represents left side. FS: Fronto-striatal; SLF: Superior longitudinal fasciculus; ILF: Inferior longitudinal fasciculus; Rt.: right; Lt.: left.

\section{Neurocognitive tests}

Clinical neuropsychological assessments were performed in all patients at the time of DTI. We used Korean version of Mini-mental Status Examination (K-MMSE) [12] for assessing global cognitive function. Attention and visuospatial working memory was assessed using digit span (DS) and visual span (VS) test. Construction ability and visuospatial memory was assessed using Rey Complex Figure Test (RFT). Leaning and verbal memory tested using Hopkins Verbal
Learning Test (HVLT) and Trail Making Test (TMT) type A for the assessment of executive function.

\section{DTI acquisition and analysis}

Diffusion tensor images were acquired using a $1.5 \mathrm{~T}$ Philips Gyroscan Intera system equipped with a Synergy-L Sensitivity Encoding (SENSE) head coil with a single-shot spin echo-planar imaging sequence. For each of the 32 non-collinear and non-coplanar diffusion-sensitizing gradients, we acquired 55 contiguous slices parallel to the anterior commissure-posterior commissure line. The imaging parameters were as follows: matrix $=128 \times 128, F O V=220 \mathrm{x}$ $220 \mathrm{~mm} 2, \mathrm{TE}=70 \mathrm{~ms}, \mathrm{TR}=12,717 \mathrm{~ms}$, SENSE factor $=2$, EPI factor $=$ $69, \mathrm{~b}=1000 \mathrm{~mm} 2 \mathrm{~s}-1$, and slice thickness $=2 \mathrm{~mm}$. We also evaluated fiber connectivity by reconstructing diffusion tensor tractography (DTT) using fiber assignment by continuous tracking (FACT) and a 3D fiber reconstruction algorithm in Philips $\mathrm{PRIDE}^{\bullet}$ software [13]. The termination criteria were fractional anisotrophy $(\mathrm{FA})<0.2$ and an angle change $>45 \mathrm{o}$, according to a previous study for the optimal trackability threshold of FA [14]. A seed ROI was drawn in each subcortical fasciculus [superior longitudinal fasciculus (SLF), inferior longitudinal fasciculus (ILF), fronto-striatal (FS) fibers] using the 2 ROI method introduced by Mori et al. [15]. The integrity of each subcortical fasciculus was measured by mean FA and apparent diffusion coefficient (ADC) values of the obtained DTT. We included diffusion metrics of the ipsilesional and contralesional subcortical fibers instead of flip it to compare lesion versus non-lesion side as we think that this might be more suitable for correlation with neuropsychological test, which are also outputs of some specialized functions of both hemisphere.

\section{Data analysis}

We used Mann-Whitney $U$ test for estimating the statistical difference of the neuropsychological test between right and left-brain lesion as well as for the comparsion of DTI metrics between ipsilesional and contralesion hemisphere with statistical significance of $\mathrm{p}<0.05$. The relationships between the neuropsychological test scores and the parameters of each subcortical fasciculus were analyzed by Spearman correlation using the SPSS package (SPSS 18.0, SPSS Inc, Chicago, IL, USA).

\section{Results}

\section{Neurocognitive test results}

Patients with left hemispheric lesions showed lower scores in MMSE and HVLT than those with lesions in the right hemisphere, while patients with right hemispheric lesions showed lower scores in TMT-A than patients with lesions in the left hemisphere; however, these differences were not statistically significant (Table 1).

\begin{tabular}{|l|l|l|l|}
\hline Neuropsychological test & $\begin{array}{l}\text { Rt. BG } \\
\text { lesion }\end{array}$ & $\begin{array}{l}\text { Lt. BG } \\
\text { lesion }\end{array}$ & p value \\
\hline K-MMSE & $25.7 \pm 3.5$ & $24.4 \pm 5.9$ & 1 \\
\hline Digit span & $8.0 \pm 4.4$ & $11.4 \pm 8.4$ & 0.73 \\
\hline Visual span & $7.3 \pm 1.2$ & $5.0 \pm 8.8$ & 0.286 \\
\hline K-HVLT & $17.7 \pm 4.9$ & $13.2 \pm 4.5$ & 0.286 \\
\hline
\end{tabular}




\begin{tabular}{|l|l|l|l|}
\hline RFT & $11.0 \pm 9.5$ & $10.7 \pm 0.4$ & 1 \\
\hline TMT-A & $53.0 \pm 45.6$ & $93.3 \pm 29.1$ & 0.393 \\
\hline
\end{tabular}

Table 1: Neuropsychological Test Scores in right and left BG lesion Patients. K-MMSE, Korean version of Mini-mental Status Examination; K-HVLT, Korean version of Hopkins Verbal Learning Test; RFT, Rey-Osterrieth Figure Test; TMT-A, Trail Making Test type A; Lt.: Left; Rt.: Right; Mann-Whitney U test ${ }^{*} \mathrm{p}<0.05$

\section{Mean FA and ADC value of subcortical fiber tracts}

We were able to reconstruct the bilateral SLF, ILF, and FS fibers using DTT in all patients except 3 in which we were unable to reconstruct their left ILF, FS and right SLF. In the reconstructed subcortical fiber tracts, the mean FA and ADC values of all fiber tracts were not significantly different in each of the fiber tracts when it was divided by lesion side (right and left hemispheric lesion) (Table 2).

\begin{tabular}{|c|c|c|c|c|c|c|c|}
\hline \multirow{2}{*}{ Tract } & \multirow{2}{*}{ Lesion } & \multicolumn{4}{|l|}{ FA ADC } & \multirow{3}{*}{$\begin{array}{l}\text { p value } \\
\text { n.s. }\end{array}$} & \multirow{3}{*}{$\begin{array}{l}\text { p value } \\
\text { n.s. }\end{array}$} \\
\hline & & \multicolumn{4}{|c|}{$\begin{array}{l}\text { Ipsilesional Contralesional } \\
\text { Ipsilesional Contralesional }\end{array}$} & & \\
\hline \multirow{2}{*}{ SLF } & Rt. & $\begin{array}{l}0.442 \pm \\
0.021\end{array}$ & $\begin{array}{l}0.431 \pm \\
0.019\end{array}$ & $\begin{array}{l}0.787 \pm \\
0.046\end{array}$ & $\begin{array}{l}0.768 \pm \\
0.052\end{array}$ & & \\
\hline & Lt. & $\begin{array}{l}0.393 \pm \\
0.070\end{array}$ & $\begin{array}{l}0.444 \pm \\
0.053\end{array}$ & $\begin{array}{l}0.885 \pm \\
0.136\end{array}$ & $\begin{array}{l}0.891 \pm \\
0.025\end{array}$ & n.s. & n.s. \\
\hline \multirow{2}{*}{ ILF } & Rt. & $\begin{array}{l}0.438 \pm \\
0.026\end{array}$ & $\begin{array}{l}0.459 \pm \\
0.014\end{array}$ & $\begin{array}{l}0.867 \pm \\
0.056\end{array}$ & $\begin{array}{l}0.878 \pm \\
0.070\end{array}$ & n.s. & n.s. \\
\hline & Lt. & $\begin{array}{l}0.406 \pm \\
0.039\end{array}$ & $\begin{array}{l}0.431 \pm \\
0.053\end{array}$ & $\begin{array}{l}0.951 \\
0.072\end{array}$ & $\begin{array}{l}1,027 \\
0.114\end{array} \pm$ & n.s. & n.s. \\
\hline \multirow{2}{*}{ FS } & Rt. & $\begin{array}{l}0.425 \pm \\
0.030\end{array}$ & $\begin{array}{l}0.432 \pm \\
0.011\end{array}$ & $\begin{array}{l}0.866 \pm \\
0.054\end{array}$ & $\begin{array}{l}0.805 \pm \\
0.056\end{array}$ & n.s. & n.s. \\
\hline & Lt. & $\begin{array}{l}0.428 \pm \\
0.034\end{array}$ & $\begin{array}{l}0.397 \pm \\
0.060\end{array}$ & $\begin{array}{l}0.875 \pm \\
0.141\end{array}$ & $\begin{array}{l}0.959 \pm \\
0.155\end{array}$ & n.s. & n.s. \\
\hline
\end{tabular}

Table 2: Diffusion metrics of the tracts in two groups according to the lesion side showing ipsilesional and contralesional FA and ADC. FA, Fractional Anisotropy; ADC, Apparent Diffusion Coefficient; SLF, Superior Longitudinal Fasciculus; ILF, Inferior Longitudinal Fasciculus; FS, Frontostriatal Fiber; BG, Basal Ganglia; Lt., Left; Rt., Right; n.s., not significant; data was shown in mean \pm standard deviation; Mann-Whitney $\mathrm{U}_{\text {test }}{ }^{*} \mathrm{p}<0.05$

\section{Relationship between cognitive function and subcortical fiber tract}

Spearman correlation analysis revealed significant findings between MMSE and DTT parameters of the left SLF: a positive correlation with FA $(\mathrm{p}<0.01)$, and a negative correlation with ADC values $(\mathrm{p}<0.01)$. Digit span and FA value of the left SLF showed a positive correlation $(\mathrm{p}<0.01)$. In the case of visual span, all subcortical fibers located in the right hemisphere showed significant correlations; the ADC values of the FS $(p<0.05)$ and the SLF $(p<0.01)$ had significant negative correlations with the visual span score. The FA $(\mathrm{p}<0.01)$ and ADC values $(\mathrm{p}<0.05)$ of the ILF also significantly correlated well with the visual span score. The RFT score showed a positive correlation with FA value $(\mathrm{p}<0.01)$ and a negative correlation with the ADC value of the right ILF $(p<0.01)$ and FS fiber $(p<0.05)$. The TMT-A score, the time to complete the task showed a significant negative correlation with the FA value $(\mathrm{p}<0.01)$ and a positive correlation with the ADC value $(p<0.01)$ of the right ILF. In addition, the TMT-A score showed a positive correlation with the ADC values of both right $(\mathrm{p}<0.05)$ and left FS fibers $(\mathrm{p}<0.05)$ (Table 3, Figure 1).

\begin{tabular}{|l|l|l|l|l|l|l|l|l|}
\hline Tract & Rt/ Lt & $\begin{array}{l}\text { DTI } \\
\text { metric } \\
\text { S }\end{array}$ & MMSE & DS & VS & HVLT & RFT & TMT-A \\
\hline & Rt. & FA & -0.29 & -0.261 & -0.206 & -0.475 & -0.086 & 0.314 \\
\hline & Rt. & ADC & -0.315 & -0.289 & $-.771^{*}$ & -0.136 & $-.712^{*}$ & $.741^{*}$ \\
\hline FS & Lt. & FA & -0.156 & -0.262 & 0.096 & 0.595 & 0.095 & -0.571 \\
\hline Liber & Lt. & ADC & -0.503 & -0.429 & -0.506 & 0.024 & -0.262 & $.872^{*}$ \\
\hline Rt. & FA & 0.126 & 0.234 & 0.543 & 0 & 0.321 & -0.214 \\
\hline Rt. & ADC & -0.613 & -0.667 & $-.898^{* *}$ & -0.321 & -0.679 & 0.692 \\
\hline LLF & Lt. & FA & $.929^{* *}$ & $.887^{* *}$ & 0.655 & 0.4 & 0.533 & -0.5 \\
\hline & Rt. & FA & $-.770^{*}$ & -0.636 & -0.511 & -0.467 & -0.35 & 0.452 \\
\hline & Rt. & ADC & -0.544 & -0.251 & $-.741^{*}$ & -0.467 & $-.850^{* *}$ & $.912^{* *}$ \\
\hline Lt. & FA & 0.381 & 0.048 & 0 & 0.333 & 0.452 & 0.036 \\
\hline & At. & ADC & -0.69 & -0.524 & -0.464 & -0.31 & -0.69 & 0.277 \\
\hline
\end{tabular}

Table 3: Correlation coefficient between cognitive function and DTI parameters of subcortical fibers; DTI: diffusion tensor imaging; FS: Fronto-striatal; SLF: Superior longitudinal fasciculus; ILF: Inferior longitudinal fasciculus; MMSE: Mini-mental Status Examination; DS: Digit Span; VS: Visual Span; HVLT: Hopkins Verbal Learning Test; RFT: Rey-Osterrieth Figure Test; TMT-A: Trail Making Test type A; Lt.: Left; Rt.: Right; FA: Fractional Anisotrophy; ADC: Apparent Diffusion Coefficient; Pearson corr: Pearson correlation. ${ }^{\star} \mathrm{p}<0.05$, ${ }^{* *}$ $\mathrm{p}<0.005$

\section{Discussion}

Cerebral infarction and recovery of patients with stroke have been a topic of intense research recently. Our results demonstrated the relationship between cognitive functions and the integrity of the adjacent subcortical fiber tracts, which were investigated by FA and ADC values of DTT in patients with basal ganglia stroke. These results showed neuroanatomical correlation with cognitive deficit in these patients. The basal ganglia is centrally located subcortical structure surrounded by subcortical fiber bundles traverse near or around the structures. The major subcortical fiber bundles of the human brain include SLF, ILF, FS fiber, inferior fronto-occipital fasciculus (IFO), uncinate fasciculus (UF), and anterior thalamic radiation (ATR) and all of theses fibers traverse near the basal ganglia, a few centimeters apart. As a result, alterations of this neural connectivity by hemorrhage or infarction of the basal ganglia can cause various remote cortical dysfunctions and produce diverse neurocognitive symptoms. Using DTI, we can analysis the anatomical integrity and make a successful identification of subcortical fiber tracts [15]. DTI provides information about the diffusion of water in tissue as a result of interactions between water in tissue and cellular structures. 
Page 4 of 5

Pathological processes that modify cell microstructures might therefore result in changes in ADC or FA. In addition, DTT methods have been developed to reconstruct connections between distant brain regions $[13,15]$. In this study, subcortical fiber tracts in the affected hemisphere showed decreased FA and increased ADC values, indicating decreased directionality and increased diffusivity due to loss of neural fibers. Deviation of both FA and ADC values are considered to reflect the degree of injury of the designated fiber track.

In our study, the MMSE scores correlated well with the connectivity of the left SLF. MMSE is a global cognitive measure thought to reflect the integrity of widely distributed cognitive networks in both hemispheres. Patients with lesions in the left hemisphere usually showed lower MMSE scores than patients with right side lesions, as this test depends on the patient's ability to understand verbal questions [16]. Our results are similar with the previous study demonstrating a strong link between MMSE score and integrity of gray matter of the left hemisphere, more than with the right hemisphere. Verbal working memory, which is measured by the digit span test also correlated well with the integrity of the left SLF. In HVLT, composed of verbal encoding and recognition processes, lower scores were shown in patients with left hemispheric lesions and mostly depended on the connectivity of the left SLF. Considering that SLF connects the frontotemporoparietal lobes, this finding is consistent with previous fMRI evidence that semantic encoding and recognition of verbal material is related with left prefrontal cortex activation $[17,18]$.

In contrast, scores assessing nonverbal memory function as measured by RFT were lower in patients with right hemispheric lesions and dependent on the connectivity of the right SLF and ILF, which interconnect the occipitoparietal lobes and the frontotemporal lobes. A previous fMRI study demonstrated activation of the right frontal and parietal lobes during the encoding and retrieval processes of RFT [19]. Disconnection of these fiber structures may contribute to deficit in the nonverbal working memory. On the other hand, TMTA, a visuospatial search task requiring rapid executive control, correlated with the integrity of the right FS fiber. The FS fiber interconnects with basal ganglia and wide range of cerebral cortex, particularly the frontal lobes. A number of functional imaging studies regarding visuospatial search tasks revealed right prefrontal and basal ganglia activation[20], which implicates that connectivity between the basal ganglia and cerebral cortex in the right hemisphere might play a role in the processing of visuospatial searches and the sequential execution.

This study has some limitations. The first is the small sample size. As this study was a preliminary study, it needs to be explored with large number of patients in the future. The second is that the sample showed average 5.9 months of stroke onset to DTI, which might have possibility to show some degenerative changes influenced to outcome, however, when one considers stroke plasticity occurring in the acute and subacute periods, it would be more accurate to obtain in the chronic period around 6 months. The third is the fact that the heterogeneity of the lesion location in the subcortical structure, which might have influenced more than cortical stroke patients as it is located in dense areas. In addition, patients with hemorrhagic stroke are usually more affected than those with ischemic stroke, which warrants further study with homogeneous group of patients.

\section{Conclusion}

Based on these results, we postulate that the neurocognitive symptoms of the patients with basal ganglia stroke depend on the integrity of adjacent subcortical fascicules, which are connected to the cortical and subcortical areas. These findings can provide an explanation for various cognitive dysfunctions in patients with basal ganglia lesion. Therefore, precise assessment of cortical-subcortical connectivity is critical for understanding clinical symptoms and establishing a successful therapeutic plan for rehabilitation

\section{Acknowlegement}

This research was supported by Basic Science Research Program through the National Research Foundation of Korea (NRF) funded by the Ministry of Education, Science and Technology (2013R1A1A2012562) and Hallym University Medical Center Research Fund (01-2012-06).

\section{References}

1. Laplane D, Levasseur M, Pillon B, Dubois B, Baulac M, et al. (1989) Obsessive-compulsive and other behavioural changes with bilateral basal ganglia lesions. A neuropsychological, magnetic resonance imaging and positron tomography study. Brain 112: 699-725.

2. Dubois B, Defontaines B, Deweer B, Malapani C, Pillon B (1995) Cognitive and behavioral changes in patients with focal lesions of the basal ganglia. Adv Neurol 65: 29-41.

3. Caplan LR, Schmahmann JD, Kase CS, Feldmann E, Baquis G, et al. (1990) Caudate infarcts. Arch Neurol 47: 133-143.

4. Kumral E, Evyapan D, Balkir K (1999) Acute caudate vascular lesions. Stroke 30: 100-108.

5. Naeser MA, Alexander MP, Helm-Estabrooks N, Levine HL, Laughlin SA, et al. (1982) Aphasia with predominantly subcortical lesion sites: description of three capsular/putaminal aphasia syndromes. Arch Neurol 39: 2-14.

6. Basser PJ, Mattiello J, LeBihan D (1994) MR diffusion tensor spectroscopy and imaging. Biophys J 66: 259-267.

7. Zhou Y, Lin FC, Zhu J, Zhuang ZG, Li YS, et al. (2008) Whole brain diffusion tensor imaging histogram analysis in vascular cognitive impairment. J Neurol Sci 268: 60-64.

8. Zhou Y, Qun-Xu, Qin LD, Qian LJ, Cao WW, et al. (2011) A primary study of diffusion tensor imaging-based histogram analysis in vascular cognitive impairment with no dementia. Clin Neurol Neurosurg 113: 92-97.

9. Lawrence AJ, Patel B, Morris RG, MacKinnon AD, Rich PM, et al. (2013) Mechanisms of cognitive impairment in cerebral small vessel disease: multimodal MRI results from the St George's cognition and neuroimaging in stroke (SCANS) study. PLoS One 8: e61014.

10. Dacosta-Aguayo R, Graña M, Fernández-Andújar M, Lopez-Cancio E, Caceres C, et al. (2014) Structural Integrity of the Contralesional Hemisphere Predicts Cognitive Impairment in Ischemic Stroke at Three Months. PLoS ONE 9: e86119.

11. Oldfield RC (1971) The assessment and analysis of handedness: the Edinburgh inventory. Neuropsychologia 9: 97-113.

12. Han C, Ahn S, Jo I, Kim E, Park MH (2008) An adaptation of the Korean mini-mental state examination (K-MMSE) in elderly Koreans: demographic influence and population-based norms (the AGE study). Arch Gerontol Geriatr 47: 302-310.

13. Mori S, Crain BJ, Chacko VP, van Zijl PC (1999) Three-dimensional tracking of axonal projections in the brain by magnetic resonance imaging. Ann Neurol 45: 265-269.

14. Kunimatsu A, Aoki S, Masutani Y, Abe O, Hayashi N, et al. (2004) The optimal trackability threshold of fractional anisotropy for diffusion 
Citation: Jung K, Jun A, Kim Y, Bashir S, Ohn SH, et al. (2015) DTI Findings Correlated with Neurocognitive Impairments in Basal Ganglia Stroke: A Preliminary Study. J Neurol Neurophysiol 6: 299. doi:10.4172/2155-9562.1000299

Page 5 of 5

tensor tractography of the corticospinal tract. Magn Reson Med Sci 3: 11-17.

15. Mori S, Kaufmann WE, Davatzikos C, Stieltjes B, Amodei L, et al. (2002) Imaging cortical association tracts in the human brain using diffusiontensor-based axonal tracking. Magn Reson Med 47: 215-223.

16. Apostolova LG, Lu PH, Rogers S, Dutton RA, Hayashi KM, et al. (2006) 3D mapping of mini-mental state examination performance in clinical and preclinical Alzheimer disease. Alzheimer Dis Assoc Disord 20: 224-231.

17. Prince SE, Daselaar SM, Cabeza R (2005) Neural correlates of relational memory: successful encoding and retrieval of semantic and perceptual associations. J Neurosci 25: 1203-1210.
18. Brassen S, Weber-Fahr W, Sommer T, Lehmbeck JT, Braus DF (2006) Hippocampal-prefrontal encoding activation predicts whether words can be successfully recalled or only recognized. Behav Brain Res 171: 271-278.

19. Engelsen BA, Gramstad A, Thomsen T, Beneventi H, Ersland L, et al. (2006) Frontoparietal activation during delayed visuospatial recall in patients with epilepsy due to hippocampal sclerosis. Epilepsy Behav 8: 565-574.

20. Fulbright RK, Manson SC, Skudlarski P, Lacadie CM, Gore JC (2003) Quantity determination and the distance effect with letters, numbers, and shapes: a functional MR imaging study of number processing. AJNR Am J Neuroradiol 24: 193-200. 